\title{
Controlling sleeping sickness - a review
}

\author{
S. C. WELBURN ${ }^{1}$, I. MAUDLIN ${ }^{1}$ and P. P. SIMARRO ${ }^{2}$ \\ ${ }^{1}$ Centre for Infectious Diseases, College of Medicine and Veterinary Medicine, The University of Edinburgh, Summerhall, \\ Edinburgh, EH9 $1 Q H$ \\ ${ }^{2}$ World Health Organization, Control of Neglected Tropical Diseases, Innovative and Intensified Disease Management, \\ Geneva, Switzerland
}

(Received 23 March 2009; revised 23 April 2009; accepted 23 April 2009; first published online 20 August 2009)

\section{SUMMARY}

Following a period characterized by severe epidemics of sleeping sickness, restoration of effective control and surveillance systems has raised the question of eliminating the disease from sub-Saharan Africa. Given sufficient political and financial support, elimination is now considered a reasonable aim in countries reporting zero or less than 100 cases per year. This success may lead health authorities across the affected region to downgrade the disease from 'neglected' to simply being ignored. In view of the significant levels of under-reporting of sleeping sickness mortality in rural communities, this could be a short-sighted policy. Loss of capacity to deal with new epidemics, which can arise as a consequence of loss of commitment or civil upheaval, would have serious consequences. The present period should be seen as a clear opportunity for public-private partnerships to develop simpler and more cost-effective tools and strategies for sustainable sleeping sickness control and surveillance, including diagnostics, treatment and vector control.

Key words: Sleeping sickness, neglected diseases, surveillance, tsetse control.

\section{IMPACT OF SLEEPING SICKNESS}

\section{A colonial disease or the colonial disease?}

The starting point for sleeping sickness control is inextricably linked to the incursion of the European powers into sub-Saharan Africa in the 19th century. Colonists hoping to profit from Africa's abundance of raw materials and cheap or even 'free' (i.e. slave) labour were disturbed to discover towards the end of that century that the workforce was succumbing in unprecedented numbers to a mysterious illness. The disease was also perceived to be spreading across the continent from the French, Portuguese and Belgian territorial interests in West and Central Africa towards the British and German colonies in East Africa. A flurry of scientific activity quickly identified trypanosomes as the causative organisms and that they were being transmitted exclusively by tsetse flies (for a detailed account of this brilliant, yet often acrimonious, period in parasitological research see the Reports of the Sleeping Sickness Commission of the Royal Society, for example, Bruce et al. 1903).

There is no doubt that 'the colonial disease' (Lyons, 1992) was responsible, in the absence of host immunity and effective drugs, for hundreds of thousands of deaths in the colonial period. We are now aware that, even with the advantage of more effective

Corresponding author: S. C. Welburn, Centre for Infectious Diseases, College of Medicine and Veterinary Medicine, The University of Edinburgh, Summerhall, Edinburgh, EH9 1QH. Tel: +44131650 6228. Fax: 44 (131)650 7348. E-mail: sue.welburn@ed.ac.uk diagnostics, data concerning sleeping sickness deaths are subject to gross errors due to under-reporting (Odiit et al. 2005). With hindsight it is reasonable to infer that millions died from sleeping sickness during the colonial period; to get some idea of the seriousness of the situation facing the colonial authorities, this pandemic could be compared to the current HIV/AIDS situation in Africa (Maudlin, 2006).

To further complicate control initiatives, it soon became apparent that sleeping sickness was not a single entity. The human disease in West and Central Africa, referred to as Gambian sleeping sickness and caused by Trypanosoma brucei gambiense, is chronic in form. Because of the chronic nature of this disease and the inaccessibility of the endemic regions it has been difficult to determine how long a patient may have been infected with $T$. brucei gambiense; recent studies have estimated that, on average, it is usually fatal within three years in the absence of treatment (Checchi et al. 2008a). Rhodesian sleeping sickness, the human disease in East and southern Africa caused by Trypanosoma brucei rhodesiense is usually fatal within 6-8 months of infection in the absence of treatment (Odiit et al. 1997; Fèvre et al. 2004). Rhodesian sleeping sickness, if left untreated, is always fatal in humans but there have always been suggestions that self-resolving and asymptomatic chronic infections of Gambian sleeping sickness may occur but it is accepted that even if they do exist they will be few in number (Checchi et al. 2008b) or misdiagnosed due to transient infections with other trypanosome subspecies (Deborggraeve et al. 2008). Critically, diagnosis and treatment of the two forms 
of sleeping sickness require differing approaches (Chappuis et al. 2005; Barrett et al. 2007).

\section{The burden of sleeping sickness}

As a result of intensive efforts to control the disease by colonial regimes using a variety of methods, there was a steady decline in the number of reported cases of sleeping sickness across Africa from the 1930s onwards. However, the post-independence period of the 1960s saw interest in, and funding for, control programmes within the endemic countries falter, resulting in a steep rise in incidence for the following thirty years. As surveillance and health care programmes in the affected regions were slowly restored following this period of neglect there has been, from 1997 onwards, a decline in reporting of new cases (Simarro et al. 2008). WHO (World Health Organization) has listed 36 countries endemic for sleeping sickness, of which 24 are affected by T. b. gambiense; seven countries (Angola, Democratic Republic of the Congo, Sudan, Chad, Central African Republic, Congo and Uganda) represent $98 \%$ of all reported Gambian sleeping sickness cases. Thirteen countries are endemic for Rhodesian sleeping sickness with Uganda and Tanzania accounting for most new cases.

1.35 million DALYs are lost due to sleeping sickness; mortality related to sleeping sickness is ranked ninth out of 25 among the human infectious and parasitic diseases in Africa (Fèvre et al. 2008a). However, in considering these data, it must be borne in mind that under-reporting is a major problem in estimating the burden of sleeping sickness. This is illustrated by the study of an outbreak of Rhodesian sleeping sickness in Uganda, which alarmingly found that $92 \%$ of sleeping sickness deaths are never reported (Odiit et al. 2005). This, despite the fact that drugs to treat sleeping sickness are provided free of charge by WHO thanks to a public-private partnership with Sanofi-Aventis. Treatment costs are high because of the supplementary drugs involved and the need for long hospitalization. It costs around US $\$ 150$ per patient (including drugs and hospitalization) to treat a Rhodesian sleeping sickness case in Uganda (Fèvre et al. 2008b) - this may be compared with the US $\$ 18$ available per capita for healthcare in Uganda (World Bank, 2006). The costs for treating Gambian sleeping sickness are similarly relatively high; for example a study of an outbreak in Democratic Republic of Congo (DRC) found sleeping sickness-related costs were equivalent to 5 months' income for affected households (Lutumba et al. 2007). We may conclude that the burden sleeping sickness imposes on rural settings is much greater than might be expected from its relative incidence.

\section{From primacy to neglect}

From being 'the Colonial disease' (Lyons, 1992) at the beginning of the 20th century, sleeping sickness is now relegated to membership of a group defined as the 'neglected tropical diseases' (NTDs), which includes leprosy, schistosomiasis, onchocerciasis, soil-transmitted helminths, lymphatic filariasis, dracunculiais, dengue, cholera, trachoma, leishmaniasis, Chagas disease and Buruli ulcer. NTDs affect several hundred million people but because they attract little attention from donors, policymakers or public health officials they are designated 'neglected' (Molyneux et al. 2005). It has been suggested that this neglect results largely from over-reliance on DALY estimates in policy planning. For most sub-Saharan African countries, accurate measurements of morbidity and mortality from neglected tropical diseases are difficult to obtain and disease burden is inevitably underestimated (King and Bertino, 2008). This insight is pertinent when considering resources for sleeping sickness which, as we have seen, is subject to gross under-reporting (Fèvre et al. 2008b) so that its true burden may not be reflected in current estimates (WHO Weekly Epidemiological Record at http:// www.who.int/wer/2006/wer8108/en/index.html).

CONTROLLING SLEEPING SICKNESS

\section{Reservoirs of disease}

Existence of a reservoir of a disease is confirmed when an infection within the target population cannot be sustained after all transmission between target and non-target populations has been eliminated (Haydon et al. 2002). The persistence of infection in a reservoir can be determined by longitudinal studies and, in the case of Rhodesian sleeping sickness, such studies were carried out exhaustively. In the Tinde experiment in Tanzania (Ashcroft, 1959), T. b. rhodesiense isolated from humans were serially transmitted by tsetse through sheep for over 20 years and remained human-infective. That wild animals could be the reservoir of Rhodesian sleeping sickness was established by injecting blood from an infected bushbuck into a human volunteer (Heisch et al. 1958). Human volunteers were later used to demonstrate that domestic animals, in this case cattle, could also act as reservoirs of Rhodesian sleeping sickness (Onyango and de Raadt, 1966). The relative epidemiological significance of wild/domestic animal reservoirs for persistence of Rhodesian sleeping sickness was not resolved until recently, when a molecular marker (SRA gene) was used to distinguish non-human infective T. . brucei from human infective T. $b$. rhodesiense in livestock (Welburn et al. 2001 a). Studies using this marker have demonstrated the overwhelming importance of the domestic animal reservoir for transmission of Rhodesian sleeping sickness in present day Uganda, with up to $40 \%$ of cattle carrying human infective trypanosomes in some endemic areas (Fèvre et al. 2001).

There have been no longitudinal studies reported for T. b. gambiense but, given the long-term nature of 
this form of disease, such experiments were probably simply considered impractical. There is evidence from studies in West Africa using PCR diagnostics that T. b. gambiense is present in both domestic and wild animals (Njiokou et al. 2006; Simo et al. 2006). However, in the absence of experiments that show T. b. gambiense is persistent in animals and animalto-human transmission, an animal reservoir for Gambian sleeping sickness cannot and perhaps need not be factored into the epidemiology of this form of the disease (Welburn et al. 2001 b). This difference in primary reservoir of disease has always, consciously or unconsciously, dictated differences in approach to controlling the two forms of sleeping sickness.

\section{Controlling Rhodesian sleeping sickness}

In controlling Rhodesian sleeping sickness, the importance of the animal reservoir was recognised by Bruce from the start of his studies in Uganda (Bruce et al. 1903). Armed with this knowledge it was but a short step for colonial regimes to advocate the destruction of wildlife and habitat that supported wildlife to control outbreaks of disease in East Africa. While this approach may have seemed logical, the ecological consequences of these methods were not thought through. The ecology of much of this region has changed considerably since colonial times and wildlife populations are much reduced in comparison with cattle forming the major reservoir of Rhodesian sleeping sickness in present-day Uganda. This change in the perception of the epidemiology of the disease has had considerable impact on control strategies. Modelling control options (whether to target the animal reservoir, humans or vector) has shown that, given the significance of the animal reservoir, vector control is by far the most efficient method for bringing outbreaks of Rhodesian sleeping sickness under control (Welburn et al. $2001 b$ ).

If we assume that vector control is the desired control option, this inevitably leads to a highly contentious issue-how best to control the fly? The technical choices are simply laid out: (1) if insecticides are the chosen route, synthetic pyrethroids are now accepted as the insecticide of choice (Allsopp and Hursey, 2004) (2) if a non-insecticide approach is chosen then the alternatives are (a) baits (traps, targets etc. with or without attractants and live baits,) or (b) the sterile insect technique (SIT). There the simplicity of tsetse control ends and more complex economic and socio-economic factors must be weighed. If the situation has already reached epidemic proportions then choices are stark and all options are open to use; in the most recent epidemic of Rhodesian sleeping sickness in Uganda in the $1980 \mathrm{~s}-1990 \mathrm{~s}$, the whole gamut of vector control methods were deployed including aerial and ground spraying of insecticides, trapping involving local communities and insecticide treated cattle (Lancien,
1991; Okoth et al. 1991; Okoth, 1999). Alongside these tsetse measures, the human reservoir was dealt with by treating those infected and attempts were made to reduce the reservoir in cattle by chemotherapy. Taken together, these methods brought the epidemic under control though the relative efficacy of these disparate approaches is not open to analysis given the security situation in the country at that time (Welburn et al. 2006). In such a serious situation, where thousands of people are infected and millions are at risk, it is understandable that every measure available is thrown at the problem. In a sense, the epidemic situation is the easier to deal with, as economics must take a back seat in such circumstances. This is in stark contrast to the endemic situation when, with low levels of mortality, health economists must weigh sleeping sickness control costs against the many other demands on their budgets and particularly the pressure to devote available resources to the 'big three' (HIV, TB and malaria), the temptation being to ignore NTDs (Molyneux, 2008). We have seen in the 20th century that the results of this neglect can be disastrous, sowing the seeds for further epidemics sooner or later. One solution would be to remove the vector altogether which could eliminate Rhodesian sleeping sickness. The Pan African Tsetse and Trypanosomosis Eradication Campaign (PATTEC) (http://www.who.int/trypanosomiasis_african/ partners/pattec/en/index.html) aims to eliminate tsetse and trypanosomiasis from Africa altogether. Isolated tsetse populations have been eliminated on Unguja Island, Zanzibar using SIT (Vreysen et al. 2000) and the Okavango Delta in Botswana by aerial spraying (Kgori et al. 2006). These approaches were not aimed at sleeping sickness but rather to remove tsetse as a threat to livestock with the economic advantages that could accrue. However, if the target tsetse population is not isolated then re-invasion makes elimination unlikely which in turn raises awkward economic and social questions (Hargrove, 2003 ; Enserink, 2007).

\section{Controlling Gambian sleeping sickness}

While removing the vector that transmits Rhodesian sleeping sickness is seen as a practical proposition on the back of programmes to control animal reservoir, the ancient foci of Gambian sleeping sickness are largely concentrated in the humid zones of west and central Africa, not suitable for the rearing of cattle and not targeted by trypanosomiasis control programmes. Modelling different control strategies showed that early case detection and treatment is most effective against Gambian infections when the involvement of an animal reservoir in the epidemiology of this form of the disease remains unresolved (Welburn et al. 2001 b). The fact that in practice active case finding has proven effective over many decades in controlling this form of the disease 
indicates that humans are the primary reservoir of T. b. gambiense (Jannin and Cattand, 2004).

There are logistical problems with active case finding in remote rural areas where health systems are weak and attendance rates at screening are often low. This approach to control relies on early diagnosis and appropriate chemotherapy, which both pose problems due to the poor sensitivity and specificity of available parasitological and serological tools but also due to the dangerous and cumbersome drugs available for treatment.

Diagnosis is reliant firstly on serological screening of the population at risk, usually by means of a Card Agglutination Test for Trypanosomiasis (CATT). Before treatment can commence clinicians would normally require parasitological confirmation of the CATT-positive test as recommended by WHO (1998); this usually involves simple microscopy or, where available, more sophisticated techniques such as the mini-anion exchange centrifugation method (Lutumba et al. 2006). Treatment of serologically positive individuals without parasitological confirmation remains controversial yet failure to respond to a CATT-positive test may have serious epidemiological consequences (Simarro et al. 1999). Staging sleeping sickness is mandatory to determine the choice of drug therapy and at present staging requires lumbar puncture to examine cerebrospinal fluid for parasites or, if parasites are not detected, CSF white blood cell count (WHO, 1998).

Chemotherapy for first (haemo-lymphatic) stage of Gambian sleeping sickness is straightforward involving a single drug, pentamidine. Treatment of the second stage, when parasites invade the central nervous system, is a much more fraught process and still largely relies on the use of melarsoprol, which is followed by a severe post-treatment reactive encephalopathy in $10 \%$ of cases, of which half die (Kennedy, 2006). Eflornithine (DFMO), a novel alternative to melarsoprol treatment (only for Gambian sleeping sickness), is safer to use (Burri and Brun, 2003; Chappuis et al. 2005) but has the major disadvantage that administration is labour intensive, requiring intravenous infusion every six hours for 14 days (56 infusions in total), a real problem for rural clinics (Priotto et al. 2008).

\section{ENDEMICITY AND CONTROL}

In countries reporting zero or less than 100 cases of sleeping sickness per year, elimination ${ }^{1}$ is now

\footnotetext{
${ }^{1}$ Elimination is the reduction to zero of the incidence of a disease in a defined geographical area as a result of deliberate efforts. Eradication is the permanent reduction to zero of the worldwide incidence of infection as a result of deliberate efforts. Elimination demands continued efforts to prevent re-establishment while intervention is no longer needed if eradication is achieved (Molyneux et al. 2004).
}

thought to be feasible given sufficient political and financial support (Simarro et al. 2008). While acknowledging the success that active surveillance measures have had from 1997 to 2006 in reducing the number of new cases of Gambian sleeping sickness in some settings, it is important to recognise that this may lead health authorities to downgrade the disease in their list of priorities. Such complacency has in the past led to a loss of capacity to deal with the disease when new epidemics arise. There must be continuing concerns about the effects which civil upheaval can have on apparently stable endemic foci (Kaba et al. 2006; Berrang Ford, 2007; Courtin et al. 2008). The important issue, as we enter a period of endemicity for sleeping sickness is: how can the 'punishment of success' (Simarro et al. loc.cit.) be best avoided?

In endemic areas, sleeping sickness cases occupy more patient admission time than all other infectious diseases excepting malaria yet their treatment has been shown to be highly cost-effective. Far from being subject to neglect, increasing coverage of therapy and reducing under-reporting of both forms of sleeping sickness should instead be a priority for health authorities (Fèvre et al. 2008a). Lutumba et al. (2007) suggest that because sleeping sickness has such a serious economic impact on households while controlling the disease is highly cost-effective, health officials should take into account the impact of sleeping sickness on families in rural Africa, rather than deferring to global statistics for disease burden (published in WHO Weekly Epidemiological Record at http://www.who.int/).

\section{Diagnostics and chemotherapy}

In the case of Gambian sleeping sickness the priority is to develop new diagnostic and chemotherapeutic tools to ease the fiscal burden of surveillance on the affected countries and hence promote and facilitate its sustainability by integrating sleeping sickness control into the normal activities of the available health systems in transmission areas. Progress is being made in detecting trypanosomes in blood and cerebro-spinal fluid based on a variety of techniques including oligochomatography (Deborggraeve et al. 2006) and isothermal tests (Njiru et al. 2008) - the first steps towards a simplified molecular test. There are also several promising developments in disease biomarkers (Papadopoulos et al. 2004; Agranoff et al. 2005). Blood or CSF markers for staging are in the early stages of development (Lejon et al. 2002; Courtioux et al. 2005) as are non-invasive diagnostic tools (Lejon et al. 2006).

A major concern for the active case finding approach to controlling Gambian sleeping sickness is the increasing number of treatment failures with melarsoprol, reportedly as high as $30 \%$ in some foci (Burri and Keiser, 2001; Stanghellini and Josenando, 2001; Robays et al. 2008). Unfortunately there are 
no new drugs forthcoming for the treatment of latestage sleeping sickness (Croft, 2008) and focus has been on trying to improve treatment regimes and combination therapies using existing drugs. Combinations of eflornithine, melarsoprol with nifurtimox (used for treatment of Chagas' disease) have been trialled in Uganda (Priotto et al. 2006) and the Congo (Priotto et al. 2007) with encouraging results.

\section{Vector control}

If elimination of tsetse is unlikely to succeed on ecological grounds or is considered too costly for the endemic situation then alternative approaches for controlling Rhodesian sleeping sickness must be considered. We now have innovative tools to kill tsetse flies, which rely less on top-down interventions but rather on the efforts of the people at risk and avoiding impositions on constrained local health budgets. Long-lasting formulations of synthetic pyrethroids that may be poured on or sprayed on cattle (Thompson et al. 1991), while effective at killing tsetse proved too expensive for poor farmers in Africa. However, it has recently been shown that it is not necessary to treat the whole animal but only the parts of cattle on which tsetse prefer to feed i.e. legs and belly (Torr et al. 2007). This restricted application technology (RAP) has reduced the cost of treatment to around US 2 cents/animal/treatment which has been shown to be affordable and well within the reach of poor farmers. Following this breakthrough in the cost of treating livestock, a public-private partnership has been formed-the Stamp Out Sleeping Sickness campaign http://www. sleepingsickness.org/) (Kabasa, 2007) - to promote the use of RAP (for vector control and to protect livestock from continued re-infection) combined with trypanocidal drugs to improve herd health and eliminate the animal reservoir of Rhodesian sleeping sickness. This campaign is aimed primarily at halting the northward spread of Rhodesian sleeping sickness to prevent it coalescing with the focus of Gambian sleeping sickness in the north west of Uganda (Picozzi et al. 2005). This approach benefits from farmers' natural desire to keep their herds healthy by reducing the burden of trypanosomiasis and also tick-borne diseases and the consequent control of the reservoir of human disease may be viewed as a very welcome public health bonus from a veterinary intervention. That farmers are willing to pay for this treatment promotes sustainability and avoids the public goods issues (Milinski et al. 2002) associated with community based trapping programmes (Brightwell et al. 2001).

\section{REFERENCES}

Agranoff, D., Stich, A., Abel, P. and Krishna, S. (2005). Proteomic fingerprinting for the diagnosis of human
African trypanosomiasis. Trends in Parasitology 21, 154-157.

Allsopp, R. and Hursey, B. S. (2004). The economics of African trypanosomiasis. In The Trypanosomiases, (ed. Maudlin, I., Holmes, P. H. and Miles, M. A.), pp. 491-507. Wallingford, UK: CABI International.

Ashcroft, M. T. (1959). The Tinde experiment. A further study of the long-term cyclical transmission of Trypanosoma rhodesiense. Annals of Tropical Medicine and Parasitology 53, 137-146.

Berrang Ford, L. (2007). Civil conflict and sleeping sickness in Africa in general and Uganda in particular. Conflict and Health 1, 6.

Barrett, M. P., Boykin, D. W., Brun, R. and Tidwell, R. R. (2007). Human African trypanosomiasis: pharmacological re-engagement with a neglected disease. British Fournal of Pharmacology 152, 1155-1171.

Brightwell, B., Dransfield, B., Maudlin, I., Stevenson, P. and Shaw, A. (2001). Reality v. Rhetoric - a Survey and Evaluation of Tsetse Control in East Africa. Agriculture and Human Values 18, 219-233.

Bruce, D., Nabarro, D. and Greig, E. D. W. (1903). Further report on sleeping sickness in Uganda. Reports of the Sleeping Sickness Commission of the Royal Society 4 , $1-87$.

Burri, C. and Brun, R. (2003). Eflornithine for treatment of human African trypanosomiasis. Parasitology Research 90 (Supp 1), S49-52.

Burri, C. and Keiser, J. (2001). Pharmacokinetic investigations in patients from northern Angola refractory to melarsoprol treatment. Tropical Medicine and International Health 6, 412-420.

Chappuis, F., Udayraj, N., Stietenroth, K., Meussen, A. and Bovier, P. A. (2005). Eflornithine is safer than melarsoprol for the treatment of second-stage Trypanosoma brucei gambiense human African trypanosomiasis. Clinical Infectious Diseases 41 , 748-751.

Checchi, F., Filipe, J. A., Barrett, M. P. and Chandramohan, D. $(2008 b)$. The natural progression of gambiense sleeping sickness: what is the evidence? PLoS Neglected Tropical Diseases 2, e303.

Checchi, F., Filipe, J. A., Haydon, D. T., Chandramohan, D. and Chappuis, F. (2008a). Estimates of the duration of the early and late stage of gambiense sleeping sickness. BMC Infectious Diseases 8, 16 .

Courtin, F., Jamonneau, V., Duvallet, G., Garcia, A., Coulibaly, B., Doumenge, J. P., Cuny, G. and Solano, P. (2008). Sleeping sickness in West Africa (1906-2006): changes in spatial repartition and lessons from the past. Tropical Medicine and International Health 13, 334-344.

Courtioux, B., Bisser, S., M'belesso, P., Ngoungou, E., Girard, M., Nangouma, A., Josenando, T., Jauberteau-Marchan, M. O. and Bouteille, B. (2005). Dot enzyme-linked immunosorbent assay for more reliable staging of patients with Human African Trypanosomiasis. Fournal of Clinical Microbiology 43, 4789-4795.

Croft, S. L. (2008). Kinetoplastida: new therapeutic strategies. Parasite 15, 522-527. 
Deborggraeve, S., Claes, F., Laurent, T., Mertens, P., Leclipteux, T., Dujardin, J. C., Herdewijn, P. and Büscher, P. (2006). Molecular dipstick test for diagnosis of sleeping sickness. Fournal of Clinical Microbiology 44, 2884-2889.

Deborggraeve, S., Koffi, M., Jamonneau, V., Bonsu, F. A., Queyson, R., Simarro, P. P., Herdewijn, P. and Büscher, P. (2008). Molecular analysis of archived blood slides reveals an atypical human Trypanosoma infection. Diagnostic microbiology and infectious disease 61, 428-433.

Enserink, M. (2007). Entomology. Welcome to Ethiopia's fly factory. Science 317, 310-313.

Fèvre, E. M., Coleman, P. G., Odiit, M., Magona, J. W., Welburn, S. C. and Woolhouse, M. E. J. (2001). The origins of a new Trypanosoma brucei rhodesiense sleeping sickness outbreak in eastern Uganda. Lancet 358, 625-628.

Fèvre, E. M., Coleman, P. G., Welburn, S. C. and Maudlin, I. (2004). Reanalyzing the 1900-1920 sleeping sickness epidemic in Uganda. Emerging Infectious Diseases 10, 567-573.

Fèvre, E. M., Odiit, M., Coleman, P. G., Woolhouse, M. E. and Welburn, S. C. $(2008 b)$. Estimating the burden of rhodesiense sleeping sickness during an outbreak in Serere, eastern Uganda. BMC Public Health 8, 96.

Fèvre, E. M., Wissmann, B. V., Welburn, S. C. and Lutumba, P. (2008a). The burden of human African trypanosomiasis. PLoS Neglected Tropical Disease 2 , e333.

Hargrove, J. W. (2003). Tsetse Eradication: Sufficiency, Necessity and Desirability. (Research Report, DFID Animal Health Programme). http://www0.sun.ac.za/ sacema/publications/TsetseEradication.html

Haydon, D. T., Cleaveland, S., Taylor, L. H. and Laurenson, M. K. (2002). Identifying reservoirs of infection: a conceptual and practical challenge. Emerging Infectious Diseases 8, 1468-1473.

Heisch, R. B., McMahon, J. P. and Manson-Bahr, P. E. C. (1958). The isolation of Trypanosoma rhodesiense from a bushbuck. British Medical Fournal ii, 1203-1204.

Jannin, J. and Cattand, P. (2004). Treatment and control of human African trypanosomiasis. Current Opinion in Infectious Diseases 17, 565-571.

Kaba, D., Dje, N. N., Courtin, F., Oke, E., Koffi, M., Garcia, A., Jamonneau, V. and Solano, P. (2006). The impact of war on the evolution of sleeping sickness in west-central Côte d'Ivoire. Tropical Medicine and International Health 11, 136-143.

Kabasa, J. D. (2007). Public-private partnership works to stamp out sleeping sickness in Uganda. Trends in Parasitology 23, 191-192.

Kennedy, P. G. (2006). Diagnostic and neuropathogenesis issues in human African trypanosomiasis. International Fournal for Parasitology 36, 505-512.

Kgori, P. M., Modo, S. and Torr, S. J. (2006). The use of aerial spraying to eliminate tsetse from the Okavango Delta of Botswana. Acta Tropica 99, 184-199.

King, C. H. and Bertino, A. M. (2008). Asymmetries of poverty: Why global burden of disease valuations underestimate the burden of neglected tropical diseases. PLoS Neglected Tropical Diseases 2, e209.
Lancien, J. (1991). Campaign against sleeping sickness in South-West Uganda by trapping tsetse flies. Annales de la Société belge de médecine tropicale. 71 (Suppl 1), 35-47.

Lejon, V., Jamonneau, V., Solano, P., Atchade, P., Mumba, D., Nkoy, N., Bébronne, N., Kibonja, T., Balharbi, F., Wierckx, A., Boelaert, M. and Büscher, P. (2006). Detection of trypanosome-specific antibodies in saliva, towards non-invasive serological diagnosis of sleeping sickness. Tropical Medicine and International Health 11, 620-627.

Lejon, V., Legros, D., Richer, M., Ruiz, J. A., Jamonneau, V., Truc, P., Doua, F., Djé, N., N'Siesi, F. X., Bisser, S., Magnus, E., Wouters, I., Konings, J., Vervoort, T., Sultan, F. and Büscher, P. (2002). IgM quantification in the cerebrospinal fluid of sleeping sickness patients by a latex card agglutination test. Tropical Medicine and International Health 7, 685-692.

Lutumba, P., Robays, J., Miaka, C., Kande, V., Mumba, D., Büscher, P., Dujardin, B. and Boelaert, M. (2006). Validity, cost and feasibility of the mAECT and CTC confirmation tests after diagnosis of African of sleeping sickness. Tropical Medicine and International Health 11, 470-478.

Lutumba, P., Makieya, E., Shaw, A., Meheus, F. and Boelaert, M. (2007). Human African trypanosomiasis in a rural community, Democratic Republic of Congo. Emerging Infectious Diseases 13, 248-254.

Lyons, M. (1992). The Colonial Disease. A Social History of Sleeping Sickness in Northern Zaire, 1900-1940. Cambridge, UK: Cambridge University Press.

Maudlin, I. (2006). African trypanosomiasis. Annnals of Tropical Medicine and Parasitology 100, 679-701.

Milinski, M., Semmann, D. and Krambeck, H. J. (2002). Reputation helps solve the 'tragedy of the commons'. Nature 415, 424-426.

Molyneux, D. H. (2008). Combating the "other diseases" of MDG 6: changing the paradigm to achieve equity and poverty reduction? Transactions of the Royal Society of Tropical Medicine and Hygiene 102, 509-519.

Molyneux, D. H., Hopkins, D. R. and Zagaria, N. (2004). Disease eradication, elimination and control: the need for accurate and consistent usage. Trends in Parasitology 20, 347-351.

Molyneux, D. H., Hotez, P. J. and Fenwick, A. (2005). "Rapid-impact interventions": How a policy of integrated control for Africa's neglected tropical diseases could benefit the poor. PLoS Medicine 2, e336.

Njiokou, F., Laveissiere, C., Simo, G., Nkinin, S., Grébaut, P., Cuny, G. and Herder, S. (2006). Wild fauna as a probable animal reservoir for Trypanosoma brucei gambiense in Cameroon. Infection, Genetics and Evolution 6, 147-153.

Njiru, Z. K., Mikosza, A. S., Matovu, E., Enyaru, J. C., Ouma, J. O., Kibona, S. N., Thompson, R. C. and Ndung'u, J. M. (2008). African trypanosomiasis : Sensitive and rapid detection of the sub-genus Trypanozoon by loop-mediated isothermal amplification (LAMP) of parasite DNA. International Fournal for Parasitology 38, 589-599.

Odiit, M., Coleman, P. G., Liu, W. C., McDermott, J. J., Fèvre, E. M., Welburn, S. C. and Woolhouse, M. E. (2005). Quantifying the level of under-detection of Trypanosoma brucei rhodesiense sleeping sickness 
cases. Tropical Medicine and International Health 10, 840-849.

Odiit, M., Kansiime, F. and Enyaru, J. C. K. (1997). Duration of symptoms and case fatality of sleeping sickness caused by Trypanosoma brucei rhodesiense in Tororo, Uganda. East African Medical fournal 74, 792-795.

Okoth, J. O. (1999). Tsetse and trypanosomiasis control problems in south-east Uganda: past, present and alternative strategies. Schweizerische medizinische Wochenschrift 129, 1091-1098.

Okoth, J. O., Okethi, V. and Ogola, A. (1991). Control of tsetse and trypanosomiasis transmission in Uganda by applications of lambda-cyhalothrin. Medical and Veterinary Entomology 5, 121-128.

Onyango, R. J., van Hoeve, K. and de Raadt, P. (1966). The epidemiology of Trypanosoma rhodesiense sleeping sickness in Alego location, central Nyanza, Kenya. I. Evidence that cattle may act as reservoir hosts of trypanosomes infective to man. Transactions of the Royal Society of Tropical Medicine and Hygiene $\mathbf{6 0}$, 175-182.

Papadopoulos, M. C., Abel, P. M., Agranoff, D., Stich, A., Tarelli, E., Bell, B. A., Planche, T., Loosemore, A., Saadoun, S., Wilkins, P. and Krishna, S. (2004). A novel and accurate diagnostic test for human African trypanosomiasis. Lancet 363, 1358-1363.

Picozzi, K., Fèvre, E. M., Odiit, M., Carrington, M., Eisler, M. C., Maudlin, I. and Welburn, S. C. (2005). Sleeping sickness in Uganda: a thin line between two fatal diseases. British Medical Fournal 331, 1238-1244.

Priotto, G., Fogg, C., Balasegaram, M., Erphas, O., Louga, A., Checchi, F., Ghabri, S. and Piola, P. (2006). Three drug combinations for late-stage Trypanosoma brucei gambiense sleeping sickness: A randomized clinical trial in Uganda. PLoS Clinical Trials 1, e39, 1-8.

Priotto, G., Kasparian, S., Ngouama, D., Ghorashian, S., Arnold, U., Ghabri, S. and Karunakara, U. (2007). Nifurtimox-eflornithine combination therapy for second-stage Trypanosoma brucei gambiense sleeping sickness : a randomized clinical trial in Congo. Clinical Infectious Diseases 45, 1435-1442.

Priotto, G., Pinoges, L., Fursa, I. B., Burke, B., Nicolay, N., Grillet, G., Hewison, C. and Balasegaram, M. (2008). Safety and effectiveness of first line eflornithine for Trypanosoma brucei gambiense sleeping sickness in Sudan: cohort study. British Medical fournal 336, 705-708.

Robays, J., Nyamowala, G., Sese, C., Betu Ku Mesu Kande, V., Lutumba, P., Van der Veken, W. and Boelaert, M. (2008). High failure rates of melarsoprol for sleeping sickness, Democratic Republic of Congo. Emerging Infectious Diseases 14, 966-967.

Simarro, P. P., Jannin, J. and Cattand, P. (2008). Eliminating human African trypanosomiasis: where do we stand and what comes next? PLoS Medicine 5, e55.

Simarro, P. P., Ruiz, J. A., Franco, J. R. and Josenando, T. (1999). Attitude towards CATT-positive individuals without parasitological confirmation in the African Trypanosomiasis (T.b.gambiense) focus of Quicama (Angola). Tropical Medicine and International Health 4, 858-861.

Simo, G., Asonganyi, T., Nkinin, S. W., Njiokou, F. and Herder, S. (2006). High prevalence of Trypanosoma brucei gambiense group 1 in pigs from the Fontem sleeping sickness focus in Cameroon. Veterinary Parasitology 139, 57-66.

Stanghellini, A. and Josenando, T. (2001). The situation of sleeping sickness in Angola: a calamity. Tropical Medicine and International Health 6, 330-334.

Thompson, J. W., Mitchell, M., Rees, R. B., Shereni, W., Scoenfield, A. H. and Wilson, A. (1991). Studies on the efficacy of deltamethrin applied to cattle for the control of tsetse flies (Glossina spp.) in southern Africa. Tropical Animal Health and Production 23, 221-226.

Torr, S. J., Maudlin, I. and Vale, G. A. (2007). Less is more: restricted application of insecticide to cattle to improve the cost and efficacy of tsetse control. Medical and Veterinary Entomology 21, 53-64.

Vreysen, M. J., Saleh, K. M., Ali, M. Y., Abdulla, A. M., Zhu, Z. R., Juma, K. G., Dyck, V. A., Msangi, A. R., Mkonyi, P. A. and Feldmann, H. U. (2000). Glossina austeni (Diptera: Glossinidae) eradicated on the island of Unguja, Zanzibar, using the sterile insect technique. Fournal of Economic Entomology 93, 123-135.

Welburn, S. C., Coleman, P. G., Maudlin, I., Fèvre, E. M., Odiit, M. and Eisler, M. C. (2006). Crisis, what crisis? Control of Rhodesian sleeping sickness. Trends in Parasitology 22, 123-128.

Welburn, S. C., Fèvre, E. M., Coleman, P. G., Odiit, M. and Maudlin, I. (2001 b). Sleeping sickness : a tale of two diseases. Trends in Parasitology 17, 19-24.

Welburn, S. C., Picozzi, K., Fèvre, E. M., Coleman, P. G., Odiit, M., Carrington, M. and Maudlin, I. (2001 a). Identification of human-infective trypanosomes in animal reservoir of sleeping sickness in Uganda by means of serum-resistance-associated (SRA) gene. Lancet 358, 2017-2019.

WHO (1998). Control and Surveillance of African Trypanosomiasis. Geneva, WHO.

World Bank Group (2006). World Development Indicators. World Bank Group Edition: 10, revised. World Bank Publications, 385 pages. 